\title{
Weight updating technique in spectrum sensing based on CAF shared diversity combining ${ }^{\dagger}$
}

\author{
Shusuke Narieda ${ }^{1 a)}$, Daiki Cho ${ }^{2 *}$, Kenta Umebayashi ${ }^{2}$, \\ and Hiroshi Naruse ${ }^{1}$ \\ ${ }^{1}$ Dept. Inform. Eng., Graduate School of Eng., Mie Univ., \\ 1577 Kurimamachiyacho, Tsu, Mie 514-8507, Japan \\ 2 Dept. Elect. and Electron. Eng., Tokyo Univ. of Agric. and Technol., \\ 2-24-16 Nakacho, Koganei, Tokyo 184-8588, Japan \\ a)narieda@pa.info.mie-u.ac.jp
}

\begin{abstract}
This paper proposes weight updating techniques for spectrum sensing based on a cyclic autocorrelation function (CAF) shared diversity combining. We had reported that CAF shared diversity combining can improve the performance by the weight calculated from the time-averaged CAF value. However, the performance is degraded when the weight includes CAFs calculated from purely additive white Gaussian noise. To avoid this, this paper proposes the weight updating technique in which only the CAFs are employed to obtain the time-averaged CAF when it is judged that a primary user is present. This paper provides theoretical analysis results of the proposed technique. The proposed results show that the performance of signal detection can be improved as compared to the conventional technique. Keywords: cognitive radio network, spectrum sensing, sequential detection, multiple antennas
\end{abstract}

Classification: Terrestrial Wireless Communication/Broadcasting Technologies

\section{References}

[1] S. Haykin, D. J. Thomson, and J. H. Reed, "Spectrum sensing for cognitive radio," Proc. IEEE, vol. 97, no. 5, pp. 849-877, May 2009. DOI:10.1109/ JPROC.2009.2015711

[2] M. Öner and F. Jondral, "Air interface recognition for a software radio system exploiting cyclostationarity," Proc. IEEE Int'l. Symp. Personal Indoor, Mobile and Radio Communications (IEEE PIMRC 2004), vol. 3, pp. 1947-1951, Sept. 2004. DOI:10.1109/PIMRC.2004.1368338

[3] K. Muraoka, M. Ariyoshi, and T. Fujii, "A robust spectrum sensing method based on maximum cyclic autocorrelation selection for dynamic spectrum access," IEICE Trans. Commun., vol. E92-B, no. 12, pp. 3635-3643, Dec. 2009. DOI:10.1587/transcom.E92.B.3635 
[4] S. Narieda, "Improved MCAS based spectrum sensing in cognitive radio," IEICE Trans. Commun., vol. E101-B, no. 3, pp. 915-923, Mar. 2018. DOI:10. 1587/transcom.2017EBP3134

[5] D. Cho, S. Narieda, K. Umebayashi, and H. Naruse, "Spectrum sensing based on weighted diversity combining using time-averaged CAF," IEICE Commun. Express, vol. 8, pp. 20-25, Feb. 2019. DOI:10.1587/comex.2018XBL0126

[6] S. M. Kay, Fundamental of Statistical Signal Processing Vol. 2, Detection Theory, Prentice-Hall, Canada, 1998.

\section{Introduction}

Frequency bands are public resources, and they must be utilized effectively in wireless communications. Cognitive radio is technology that can realize the effective utilization of frequency bands. Spectrum sensing techniques are employed to seek a spatial and temporal vacancy frequency bands, and it is important for the cognitive radio networks [1]. Various feature detection, which is referred to as cyclostationary detection, can execute signal detection without the measurement noise floor have been reported $[2,3,4,5]$. Among these techniques, the technique [5] can obtain a good sensing performance with a low computational complexity. To improve the performance, this technique employs a weight factor that is obtained by a time-averaged cyclic autocorrelation function (CAF). The technique extracts the information of channel gain by time-averaged CAFs, and it needs to gather the CAF having the information of channel gain. However, the conventional technique uses the weight factor obtained from both the CAF of received signals and purely AWGN. To solve the problem, this paper proposes a weight updating technique for statistics shared CAF diversity combining based spectrum sensing.

\section{Preliminary notion}

\subsection{Spectrum sensing}

We consider the spectrum sensing of primary user's (PU's) orthogonal frequency division multiplexing (OFDM) signals that are composed of samples for a useful symbol duration (a fast Fourier transform (FFT)) $N_{\mathrm{FFT}}$ and cyclic prefix (CP) duration $N_{\mathrm{CP}}$, and the OFDM symbol duration $N_{\mathrm{OFDM}}\left(N_{\mathrm{OFDM}}=N_{\mathrm{FFT}}+N_{\mathrm{CP}}\right)$ at secondary users (SUs) with $N_{\mathrm{R}}$ received antennas and RF chains. We let $\mathcal{H}_{1}$ and $\mathcal{H}_{0}$ denote the hypotheses in which the PU is active and inactive, respectively. Further, the received signal at $i$ th receive antenna $r_{i}(n)$ is determined by using a binary hypothesis testing problem [6] as

$$
\begin{aligned}
& \mathcal{H}_{1}: r_{i}(n)=h_{i} s(n)+v_{i}(n) \\
& \mathcal{H}_{0}: r_{i}(n)=v_{i}(n)
\end{aligned}
$$

where $h_{i}, s(n)$ and $v_{i}(n)$ are a channel coefficient, a PU's OFDM signal and AWGN respectively, and the subscript $(\cdot)_{i}$ represents the receive antenna index. Moreover, $v_{i}(n)$ follows a circularly symmetric complex Gaussian, and $v_{i}(n) \sim \mathcal{C N}\left(0, \sigma_{v}^{2} / 2\right)$. $h_{i}, s(n)$ and $v_{i}(n)$ are identically, independent distributions to each other. 


\subsection{Weighted CAF shared diversity combining}

In the spectrum sensing technique based on multiple receive antennas, the computational complexity for signal detection increases as the number of receive antenna increases. This is because the statistics are computed from received signals obtained from each receive antenna. To reduce the complexity so as not to degrade the performance of signal detection, we proposed weighted CAF shared diversity combining techniques [5]. In this technique, two statistics $\mathcal{T}_{\alpha_{1}}(m N)$ and $\mathcal{T}_{\beta_{k}}(m N)$, which are based on the CAFs at cyclic frequencies $\alpha\left(\alpha=1 / N_{\text {OFDM }}\right)$ and $\beta_{k}$ $\left(\beta_{k}=(k+0.5) / N_{\text {OFDM }}\right)$ respectively, are computed. These can be written as

$$
\begin{aligned}
& \mathcal{T}_{\alpha_{1}}(m N)=\left|\sum_{i=1}^{N_{\mathrm{R}}} w_{i}^{\left(\alpha_{1}\right)}(m N) \hat{R}_{r_{i}, N}^{\alpha_{1}}(m N)\right| \\
& \mathcal{T}_{\beta_{k}}(m N)=\left|\hat{R}_{r_{\mathcal{I}}, N^{\prime}}^{\beta_{k}}(m N)\right|, \quad k=0, \cdots, N_{\mathrm{D}}-1,
\end{aligned}
$$

where $N, N^{\prime}, N_{\mathrm{D}}, w_{i}^{\left(\alpha_{1}\right)}(m N), \hat{R}_{r_{i}, N}^{\alpha_{1}}(m N), \mathcal{I}$ and $\hat{R}_{r_{I}, N^{\prime}}^{\beta_{k}}(m N)$ are the number of samples to compute the CAF at $\alpha_{1}$, the number of samples to compute the CAF at $\beta_{k}\left(k=0, \cdots, N_{\mathrm{D}}-1\right)$, the number of CAFs at $\beta_{k}$, a weight factor that can be obtained using a time-averaged CAF $\mathcal{R}_{N_{\mathrm{T}}}^{(i)}(t N)$, the approximated CAF at $\alpha_{1}$ computed by $r_{i}(n)$ for $N$ samples, an arbitrary integer and $1 \leq \mathcal{I} \leq N_{\mathrm{R}}$ and the approximated CAF at $\beta_{k}$ computed by $r_{\mathcal{I}}(n)$ for $N^{\prime}$ samples, respectively. $\hat{R}_{r_{i}, N}^{\alpha_{1}}(m N)$ and $\hat{R}_{r_{T}, N^{\prime}}^{\beta_{k}}(m N)$ are given by

$$
\begin{aligned}
& \hat{R}_{r_{i}, N}^{\alpha_{1}}(m N)=\frac{1}{N} \sum_{n=m N-N+1}^{m N} r_{i}(n) r_{i}\left(n+N_{\mathrm{FFT}}\right) e^{-j 2 \pi \alpha_{1} n \Delta t}, \quad i=1, \cdots, N_{\mathrm{R}} \\
& \hat{R}_{r_{I}, N^{\prime}}^{\beta_{k}}(m N)=\frac{1}{N^{\prime}} \sum_{n=m N-(k+1) N^{\prime}+1}^{m N-k N^{\prime}} r_{\mathcal{I}}(n) r_{\mathcal{I}}\left(n+N_{\mathrm{FFT}}\right) e^{-j 2 \pi \beta_{k} n \Delta t}, \quad k=0, \cdots, N_{\mathrm{D}}-1
\end{aligned}
$$

where $\Delta t$ is a sampling interval. Further, $\overline{P_{\mathrm{FA}}}=1 /\left(N_{\mathrm{D}}+1\right)$ where $\overline{P_{\mathrm{FA}}}$ is a target false alarm probability. $w_{i}^{\left(\alpha_{1}\right)}(m N)$ and $\mathcal{R}_{N_{\mathrm{T}}}^{(i)}(t N)$ are given by respectively as

$$
\begin{aligned}
& w_{i}^{\left(\alpha_{1}\right)}(m N)=\frac{\left|\mathcal{R}_{N_{\mathrm{T}}}^{(i)}([m-1] N)\right|}{\sqrt{\sum_{q=1}^{N_{\mathrm{R}}}\left|\mathcal{R}_{N_{\mathrm{T}}}^{(q)}([m-1] N)\right|^{2}}}, \quad i=1, \cdots, N_{\mathrm{R}} \\
& \mathcal{R}_{N_{\mathrm{T}}}^{(i)}(m N)=\frac{1}{N_{\mathrm{T}}} \sum_{t=m-N_{\mathrm{T}}+1}^{m} \hat{R}_{r_{i}, N}^{\alpha_{1}}(t N), \quad i=1, \cdots, N_{\mathrm{R}},
\end{aligned}
$$

where $N_{\mathrm{T}}$ is the number of $\hat{R}_{r_{i}, N}^{\alpha_{1}}(m N)$ used for the time-averaged computation. From these, a final judgment can be obtained as

$$
\mathcal{T}_{\alpha_{1}}(m N) \stackrel{\mathcal{H}_{1}}{\underset{\mathcal{H}_{0}}{\gtrless}} \sqrt{\frac{N^{\prime}}{N}} \max _{k}\left[\mathcal{T}_{\beta_{k}}(m N)\right],
$$

where $\max _{k}\left[\mathcal{X}_{k}\right]$ is a maximum value of $\mathcal{X}_{k}$ for $k$.

\section{Weight updating techniques and its analyses}

The CAF shared diversity combining extracts the information of channel gain by averaging computed CAFs, and it requires gathering the CAFs that hold such information. Therefore, this paper proposes the weight updating technique for the CAF shared diversity combining. The proposed technique employs the CAF having 
information regarding channel gain for the computation of the time-averaged weight factor. Concretely, when the final judgment of signal detection is true $\left(\mathcal{H}_{1}\right)$, the weight factor is computed. From these, we let $\overline{w_{i}^{\left(\alpha_{1}\right)}}\left(q_{\gamma}\right)$ and $\overline{\mathcal{R}_{N_{\mathrm{T}}}^{(i)}}\left(q_{\gamma}\right)$ be newly defined as a weight factor and time-averaged CAF at $q_{\gamma}\left(q_{\gamma}>q_{\gamma-1}\right)$ respectively, as

$$
\begin{aligned}
& \overline{w_{i}^{\left(\alpha_{1}\right)}}\left(q_{\gamma}\right)=\frac{\left|\overline{\mathcal{R}_{N_{\mathrm{T}}}^{(i)}}\left(q_{\gamma-1}\right)\right|}{\sqrt{\sum_{l=1}^{N_{\mathrm{R}}}\left|\overline{\mathcal{R}_{N_{\mathrm{T}}^{(l)}}^{(l)}}\left(q_{\gamma-1}\right)\right|^{2}}}, \quad i=1, \cdots, N_{\mathrm{R}} \\
& \overline{\mathcal{R}_{N_{\mathrm{T}}}^{(i)}}\left(q_{\gamma}\right)=\frac{1}{N_{\mathrm{T}}} \sum_{p=1}^{N_{\mathrm{T}}} \hat{R}_{r_{i}, N}^{\alpha_{1}}\left(u_{p, q_{\gamma}} \mid \mathcal{H}_{1}\right), \quad i=1, \cdots, N_{\mathrm{R}},
\end{aligned}
$$

where $\hat{R}_{r_{i}, N}^{\alpha_{1}}\left(u_{p, q_{\gamma}} \mid \mathcal{H}_{1}\right)$ is a newly defined $\hat{R}_{r_{i}, N}^{\alpha_{1}}(t N)$ when the final judgment is true $\left(\mathcal{H}_{1}\right) \cdot u_{p, q_{\gamma}}$ is the time in which the $p$ th $\mathrm{CAF}$ for $\overline{\mathcal{R}_{N_{\mathrm{T}}}^{(i)}}\left(q_{\gamma}\right)$ is obtained, and it is given by

$$
\begin{aligned}
u_{p, q_{\gamma}}= & \left\{n \mid \text { final judgment is true }\left(\mathcal{H}_{1}\right)\right\}, \\
& p=1, \cdots, N_{\mathrm{T}}, \quad q_{\gamma}>u_{N_{\mathrm{T}}, u_{p, q_{\gamma}}}>u_{N_{\mathrm{T}}-1, u_{p, q_{\gamma}}}>\cdots>u_{1, u_{p, q_{\gamma}}} \geq q_{\gamma-1} .
\end{aligned}
$$

Next, the effect of the technique is theoretically proved. We let $\Psi$ denote a channel occupancy ratio which is the probability of the target signal occupancies in the channel to be monitored by SUs, and $0 \leq \Psi \leq 1$. Further, we let $\xi$ denote the probability that the final judgment is true $\left(\mathcal{H}_{1}\right)$ whether or not the target signal is included in the CAF. Note that $\xi$ also represents the probability the weight factor is updated in the proposed technique. $\xi$ can be represented by

$$
\xi=\Psi P_{\mathrm{D}}+(1-\Psi) P_{\mathrm{FA}} .
$$

Note that the first and second terms of eq. (12) are the probabilities that the SU can detect the OFDM signals and the false alarm probability at the SUs, respectively. Based on $\xi$, we show the effect of the proposed technique. We let $\lambda_{\mathrm{P}}\left(\mathcal{H}_{1} \mid \mathcal{H}_{1}\right)$ and $\lambda_{\mathrm{P}}\left(\mathcal{H}_{1} \mid \mathcal{H}_{0}\right)$ denote the conditional probability whether the target signal is included in the CAF or not, respectively. $\lambda_{\mathrm{P}}\left(\mathcal{H}_{1} \mid \mathcal{H}_{1}\right)$ and $\lambda_{\mathrm{P}}\left(\mathcal{H}_{1} \mid \mathcal{H}_{0}\right)$ are given by

$$
\begin{aligned}
& \lambda_{\mathrm{P}}\left(\mathcal{H}_{1} \mid \mathcal{H}_{1}\right)=\frac{\Psi P_{\mathrm{D}}}{\Psi P_{\mathrm{D}}+(1-\Psi) P_{\mathrm{FA}}} \\
& \lambda_{\mathrm{P}}\left(\mathcal{H}_{1} \mid \mathcal{H}_{0}\right)=\frac{(1-\Psi) P_{\mathrm{FA}}}{\Psi P_{\mathrm{D}}+(1-\Psi) P_{\mathrm{FA}}} .
\end{aligned}
$$

Furthermore, we let $\lambda_{\mathrm{C}}\left(\mathcal{H}_{1}\right)$ and $\lambda_{\mathrm{C}}\left(\mathcal{H}_{0}\right)$ denote the probability where the target signal is included in the $\mathrm{CAF}$ or not in the conventional technique [5], respectively. Because the weight factor is always updated when the CAF is computed whether or not the target signal is included in the CAF, these can be written as

$$
\begin{aligned}
& \lambda_{\mathrm{C}}\left(\mathcal{H}_{1}\right)=\Psi \\
& \lambda_{\mathrm{C}}\left(\mathcal{H}_{0}\right)=1-\Psi .
\end{aligned}
$$

Here, we compare $\lambda_{\mathrm{P}}\left(\mathcal{H}_{1} \mid \mathcal{H}_{1}\right)$ and $\lambda_{\mathrm{C}}\left(\mathcal{H}_{1}\right)$, i.e., we derive $\lambda_{\mathrm{P}}\left(\mathcal{H}_{1} \mid \mathcal{H}_{1}\right)-\lambda_{\mathrm{C}}\left(\mathcal{H}_{1}\right)$ as

$$
\lambda_{\mathrm{P}}\left(\mathcal{H}_{1} \mid \mathcal{H}_{1}\right)-\lambda_{\mathrm{C}}\left(\mathcal{H}_{1}\right)=\left(P_{\mathrm{D}}-P_{\mathrm{FA}}\right) \frac{(1-\Psi) \Psi}{\Psi P_{\mathrm{D}}+(1-\Psi) P_{\mathrm{FA}}} .
$$




$$
\lambda_{\mathrm{P}}\left(\mathcal{H}_{1} \mid \mathcal{H}_{0}\right)-\lambda_{\mathrm{C}}\left(\mathcal{H}_{0}\right)=-\left(P_{\mathrm{D}}-P_{\mathrm{FA}}\right) \frac{\Psi}{\Psi P_{\mathrm{D}}+(1-\Psi) P_{\mathrm{FA}}} .
$$

Similar to eq. (17), $\lambda_{\mathrm{P}}\left(\mathcal{H}_{1} \mid \mathcal{H}_{0}\right) \leq \lambda_{\mathrm{C}}\left(\mathcal{H}_{0}\right)$ can always be achieved because of $P_{\mathrm{D}} \geq P_{\mathrm{FA}}$. From these, it can be said that the weight factor of the proposed technique contains a lot of CAFs of target signals than that of the conventional one.

\section{Numerical example}

\subsection{Parameter setup}

In order to validate the effectiveness of the proposed technique, some numerical examples are shown in this section. The target signal is OFDM, and on each subcarrier, data symbols are modulated with quadrature phase shift keying. The number of subcarriers (or the number of FFT points) is 64 . The length of the CP is a quarter of the length of the OFDM symbols, i.e., 16 $t$. A Rayleigh flat fading channel model is employed. The target false alarm probability $\overline{P_{\mathrm{FA}}}$ is set to 0.1 , and the number of cyclic frequencies $\beta_{k}$ for decision $N_{\mathrm{D}}$ is set to 9 to realize $\overline{P_{\mathrm{FA}}}=0.1$. The number of samples $N$ and $N^{\prime}$ for the CAF computation are 2560 and 268, respectively. The number of receive antennas $N_{\mathrm{R}}$ is 2,4 , and 8 . We employ two-state Markov model to generate the traffic pattern. In the model, $g_{i j}$ is a probability which transits from the state $\mathcal{H}_{i}$ to the state $\mathcal{H}_{j}$. It is known that $g_{01}=$ $g_{10} \Psi /(1-\Psi), g_{11}=1-g_{10}$ and $g_{00}=1-g_{01}$. Further, we employ $\Psi=0.1$, $0.2,0.3$ and $g_{10}=0.25$. Results shown in this section are obtained after $N_{\mathrm{T}}$ CAFs with the decision $\mathcal{H}_{1}$ are collected.

\subsection{Performance comparison}

First, we evaluate the performance of false alarm probability for the proposed technique and conventional technique. Note that results of false alarm probability are obtained when only AWGN is included in the received signals for $\Psi \neq 0$. Table I shows the performance of false alarm probability for the proposed technique and conventional technique when $N_{\mathrm{R}}=2,4,8$ and $\Psi=0.2$. As shown in Table I, the performances of both techniques are almost the same. Next, we evaluate the performance of signal detection probability for both techniques. Fig. 1 shows the performance of signal detection probability of the proposed technique and conventional technique for $N_{\mathrm{R}}=2,4,8, N_{\mathrm{T}}=10,20,50$ and $\Psi=0.1,0.2,0.3$. As shown in Figs. $1 \mathrm{a}, 1 \mathrm{~b}$ and $1 \mathrm{c}$, it can be seen that the performance of the proposed technique outperforms that of the conventional

Table I. False alarm probability for proposed technique and conventional technique. $\overline{P_{\mathrm{FA}}}=0.1, N_{\mathrm{R}}=2,4,8$ and $\Psi=0.2$.

\begin{tabular}{c|cc|cc|cc}
\hline$N_{\mathrm{R}}$ & \multicolumn{2}{|c|}{2} & \multicolumn{2}{c|}{4} & \multicolumn{2}{c}{8} \\
\hline$N_{\mathrm{T}}$ & Proposed & Conventional & Proposed & Conventional & Proposed & Conventional \\
\hline 5 & 0.1003 & 0.1003 & 0.0998 & 0.0995 & 0.0998 & 0.0999 \\
10 & 0.0999 & 0.0998 & 0.0997 & 0.0996 & 0.1002 & 0.1007 \\
20 & 0.0994 & 0.0993 & 0.0999 & 0.1002 & 0.1003 & 0.1002 \\
50 & 0.0992 & 0.0992 & 0.0994 & 0.0996 & 0.0991 & 0.0989 \\
\hline
\end{tabular}




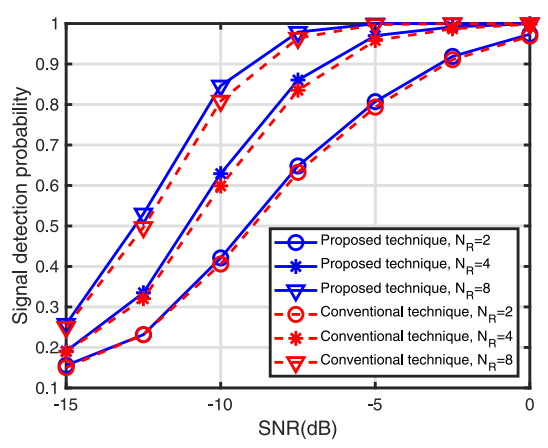

(a) $N_{\mathrm{T}}=10$ and $\Psi=0.1$.

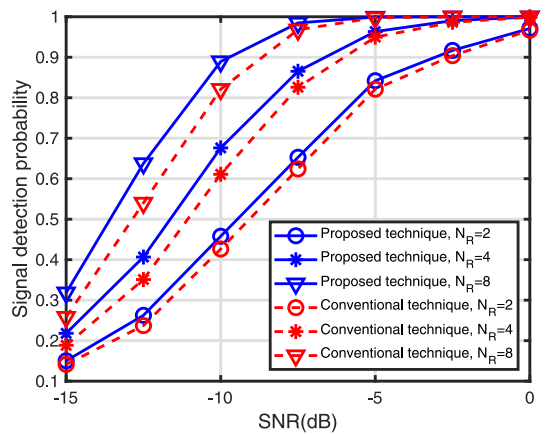

(c) $N_{\mathrm{T}}=50$ and $\Psi=0.1$.

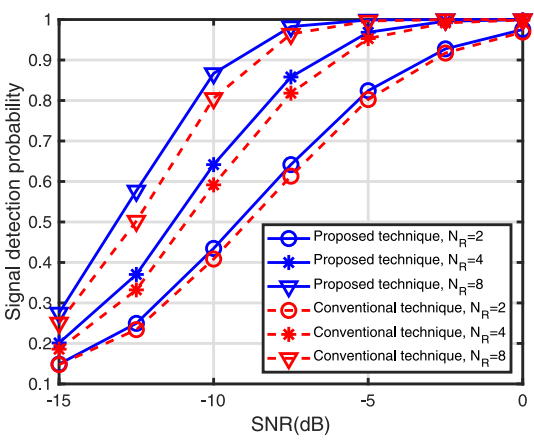

(b) $N_{\mathrm{T}}=20$ and $\Psi=0.1$.

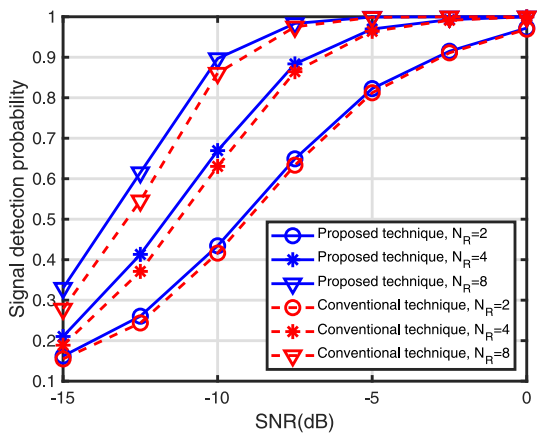

(d) $N_{\mathrm{T}}=50$ and $\Psi=0.2$.

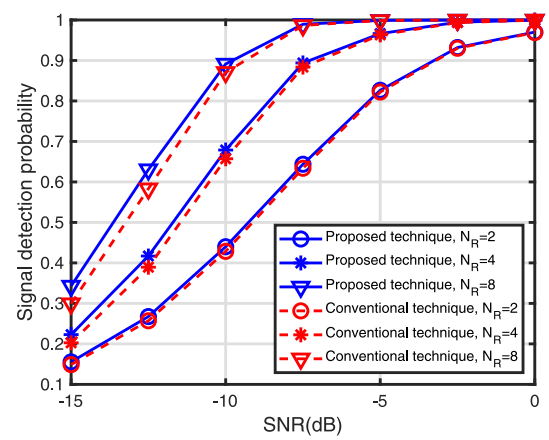

(e) $N_{\mathrm{T}}=50$ and $\Psi=0.3$.

Fig. 1. Signal detection probability for proposed technique and conventional technique. $\Psi=0.1,0.2,0.3, N_{\mathrm{R}}=2,4,8$ and $N_{\mathrm{T}}=$ $10,20,50$.

technique as $N_{\mathrm{T}}$ and $N_{\mathrm{R}}$ increases. Further, as shown in Figs. 1c, 1d and 1e, it can be seen that the proposed technique is effective when $\Psi$ is low.

\section{Conclusion}

This paper proposed a weight updating technique for the statistics shared CAF diversity combining based spectrum sensing. In this paper, the time-averaged CAF is obtained by exclusively employing only the those CAFs when the PU is present. Further, this paper provided the theoretical analyses of the proposed technique. The proposed results showed that the performance of signal detection is improved as compared to that of the conventional technique.

\section{Acknowledgment}

This work was supported by JSPS KAKENHI Grant Number JP19K04374. 